\title{
Magnifying endoscopy with narrow-band imaging for gastric heterotopic pancreas
}

\section{다 (용}

\author{
Authors \\ Heetaek Oh, Gwang Ha Kim, Moon Won Lee, Hye Kyung Jeon, Dong Hoon Baek, Bong Eun Lee
}

Institution

Department of Internal Medicine, Pusan National University

School of Medicine and Biomedical Research Institute,

Pusan National University Hospital, Busan, Korea

submitted 5.10.2017

accepted after revision $\quad$ 18.12.2017

Bibliography

DOI https://doi.org/10.1055/s-0044-101350 |

Endoscopy International Open 2018; 06: E369-E375

(c) Georg Thieme Verlag KG Stuttgart · New York

ISSN 2364-3722

Corresponding author

Gwang Ha Kim, MD, PhD, Department of Internal Medicine,

Pusan National University School of Medicine and

Biomedical Research Institute, Pusan National University

Hospital, 179 Gudeok-ro, Seo-Gu, Busan 49241, Korea

Fax: +82-51-244-8180

doc0224@pusan.ac.kr

\section{ABSTRACT}

Background and study aims Heterotopic pancreas is a common subepithelial lesion in the stomach. However, its histological diagnosis is difficult when tissue samples are obtained with a conventional biopsy forceps. This study aimed to describe the magnifying endoscopy with narrowband imaging (ME-NBI) features of gastric heterotopic pancreas.
Patients and methods We retrospectively analyzed a database of all patients who underwent endoscopic ultrasonography (EUS) at Pusan National University Hospital from January 2010 to December 2010. Thirty-six patients with endosonographically diagnosed heterotopic pancreas who underwent ME-NBI and endoscopic ultrasonography (EUS) simultaneously were studied. The ME-NBI features of their lesions were analyzed.

Results Thirty lesions were located in the antrum and six in the body. Six lesions (17\%) showed umbilication or central dimpling on the surface, and nine (25\%) had a macroscopic opening on the surface. On ME-NBI, a microscopic opening was identified in 22 (81\%) of 27 lesions wherein a macroscopic opening was not observed during conventional endoscopy. Macroscopic or microscopic opening was observed in 31 lesions ( $86 \%$ ). The frequency of macroscopic or microscopic opening was higher in lesions with anechoic duct-like structures than in lesions without such structures on EUS ( $91 \%$ [29/32] vs $50 \%$ [2/4], $P=0.027)$. Focal loss of microsurface structure and presence of a thickened submucosal vessel were observed in $6(17 \%)$ and 5 lesions (14\%), respectively.

Conclusions The characteristic ME-NBI feature of heterotopic pancreas is presence of a microscopic opening on its surface. This ME-NBI feature is potentially useful for differentiating heterotopic pancreas from other gastric subepithelial tumors.

\section{Introduction}

Heterotopic pancreas, also called aberrant pancreas, is defined as pancreatic tissue that lacks anatomic or vascular continuity with the normal pancreas. It is noted in $0.6 \%$ to $13 \%$ of autopsies and is also found in approximately 1 in every 500 surgeries involving the upper abdomen [1]. Heterotopic pancreas is abnormally located in other organs and is mostly found in the upper gastrointestinal tract adjacent to the pancreas; in $90 \%$ of the patients with heterotopic pancreas, it is commonly located in the stomach, duodenum, or proximal part of the jejunum [2].
Histopathologic confirmation of heterotopic pancreas is usually not possible for two reasons [3]. First, tissue specimens obtained using a standard endoscopic biopsy forceps are not adequate for histopathological diagnosis of heterotopic pancreas. Second, endoscopic or surgical resection is usually unnecessary for most asymptomatic patients. However, differentiating heterotopic pancreas from other mesenchymal tumors, particularly gastrointestinal stromal tumors, is often difficult due to nonspecific endoscopic findings [4]. Endoscopic ultrasonography (EUS) is the most helpful diagnostic modality to distinguish subepithelial lesions in the gastrointestinal tract. We 

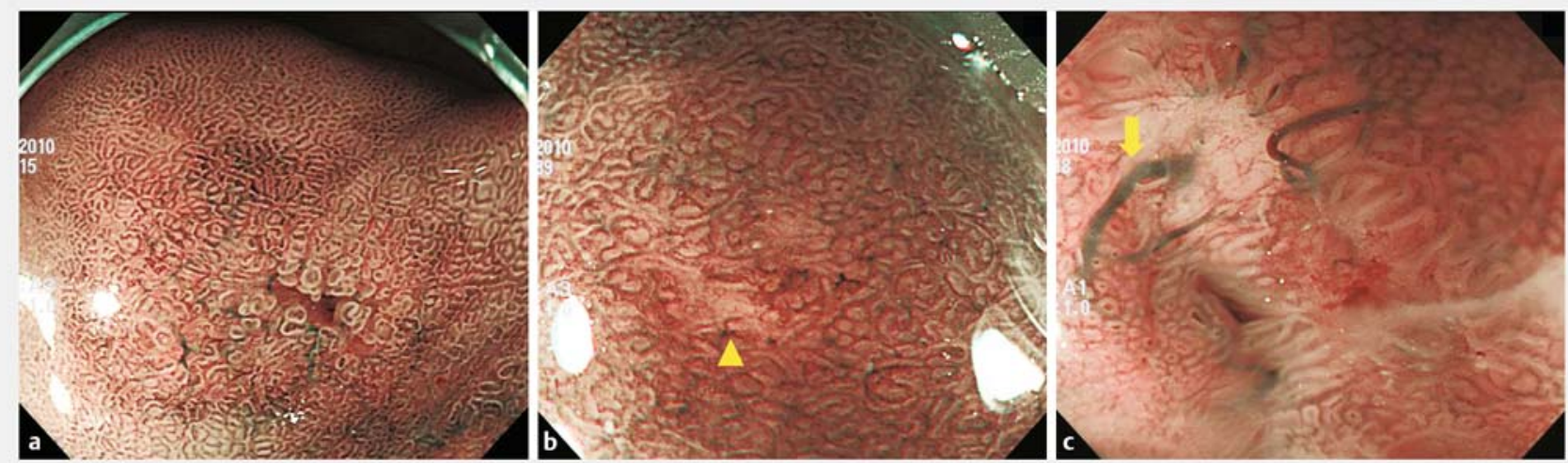

- Fig. 1 Magnifying endoscopy with narrow-band imaging for gastric heterotopic pancreas. a Presence of a microscopic opening on the surface. b Focal loss of microsurface structures (arrowhead). c Presence of a thickened submucosal vessel (arrow).

previously reported the usefulness of EUS in diagnosing heterotopic pancreas in the stomach [5]. The characteristic EUS features of heterotopic pancreas include indistinct borders, lobulated margins, presence of anechoic duct-like structures, a mural growth pattern, and localization within two or more layers.

Histologically, heterotopic pancreas mainly consists of exocrine tissue (acini) and excretory ducts. The small ducts sometimes form a common large duct, but sometimes drain directly into the gastric lumen. Therefore, drainage of ducts of heterotopic pancreas into the gastric lumen suggests presence of an opening on the surface of the heterotopic pancreas. Although umbilication or central dimpling on the surface strongly suggests the opening of a large excretory duct, this finding is observed in fewer than one-third of cases of heterotopic pancreas [5].

Magnifying endoscopy with narrow-band imaging (ME-NBI) is a useful modality to visualize in detail the microstructures and microvessels within the superficial layer of the gastric mucosa. Thus, the site where the duct of heterotopic pancreas drains in the gastric lumen could be visualized using ME-NBI. To date, no report has addressed the ME-NBI findings of gastric heterotopic pancreas. Therefore, the aim of this study was to describe the ME-NBI features of gastric heterotopic pancreas.

\section{Patients and methods}

We reviewed a prospectively maintained single-center EUS database of all 364 patients who underwent EUS for a gastric subepithelial lesion at Pusan National University Hospital (Busan, Korea) from January 2010 to December 2010. A total of 57 patients with endosonographically or histologically diagnosed heterotopic pancreas were identified. Of them, 36 patients who underwent both ME-NBI and EUS at the same time were included in this study. A retrospective review was performed to obtain patient demographics, imaging (EUS, ME-NBI), and pathology. This study was reviewed and approved by the Institutional Review Board at Pusan National University Hospital (ap- proval number $\mathrm{H}-1710-009-059)$, and informed consent was obtained from all patients.

\section{Magnifying endoscopy with narrow-band imaging}

The video endoscopy system used was the EVIS-LUCERA SPECTRUM system (Olympus, Tokyo, Japan), which consisted of a light source (CLV-260SL), a processor (CV-260SL), and a magnifying video endoscope (GIF-H260Z). To obtain a clear view for $\mathrm{ME}-\mathrm{NBI}$, a soft hood (MB-46; Olympus) was fitted on the distal tip of the endoscope to maintain the focal distance. ME-NBI was performed by a single experienced endoscopist (GHK) who had previously performed more than 100 ME-NBI examinations. All examinations were performed under conscious sedation with 2 to $5 \mathrm{mg}$ of midazolam. During conventional endoscopy for subepithelial lesions, the following endoscopic features were prospectively recorded for all lesions: (1) location; (2) macroscopic shape (Yamada classification [6]); and (3) presence of central dimpling, umbilication, or opening on the surface. Subsequently, ME-NBI was performed; during ME-NBI, presence of a microscopic opening on the surface, the status of microsurface structure, and presence of a thickened submucosal vessel were prospectively evaluated ( $\triangleright$ Fig. 1 ).

\section{Endoscopic ultrasonography}

EUS was performed by an experienced endosonographer (GHK) using a radial scanning echoendoscope (GF-UM2000; Olympus) with variable frequencies of $7.5,12$, and $20 \mathrm{MHz}$, as well as a 20$\mathrm{MHz}$ radial scanning catheter probe (UM3D-DP20 - 25R; Olympus) [5]. Scanning of the lesion was carried out after filling the stomach with 400 to $800 \mathrm{~mL}$ of deaerated water. The following EUS features were prospectively recorded for all lesions: (1) maximal diameter; (2) growth pattern (intraluminal, mural, or extraluminal); (3) sonographic layer of origin; (4) echogenicity (hypoechoic or hyperechoic); (5) homogeneity (homogenous or heterogeneous); (6) distinctness of the border (distinct or indistinct); and (7) presence of anechoic duct-like structures. Heterotopic pancreas was endosonographically diagnosed if a subepithelial tumor had typical EUS features such as an indis- 
tinct border, presence of anechoic duct-like structures, a mural growth pattern, and localization within two or more layers [5].

Based on the sonographic layer of origin, we endosonographically classified heterotopic pancreas into two types, namely, superficial type (S-type) and deep type (D-type) [5]. S-type lesions originated in the second (deep mucosal) and/or the third (submucosal) layers, and D-type lesions originated in the third (submucosal) and the fourth (muscularis propria) layers with or without extension into the fifth (subserosal or serosal) layer.

\section{Statistical analysis}

Variables are expressed as medians or ranges and simple proportions. Statistical significance of differences in the frequency of macroscopic or microscopic opening according to the type of heterotopic pancreas and the presence of anechoic ductlike structures on EUS was assessed using the $x^{2}$ test. A $P$-value $<0.05$ was considered statistically significant. Statistical calculations were performed using IBM SPSS version 23.0 for Windows (IBM Co., Armonk, NY).

\section{Results}

A total of 36 patients (22 men and 14 women) aged 15 to 70 years (median age: 40 years) were included in the study. Thirty lesions were located in the antrum and 6 lesions were in the body ( $\triangleright$ Table 1$)$. All lesions had Yamada type I macroscopic shape and 6 lesions $(6 / 36,17 \%)$ showed umbilication or central dimpling on the surface. Macroscopically, an opening was observed in nine lesions (9/36, 25\%). Bite-on-bite biopsy was performed in 23 lesions. Of these 23 lesions, five were histopathologically diagnosed as heterotopic pancreas.

As shown by EUS, the lesions were mainly located in the second (deep mucosal), third (submucosal), and/or fourth (muscularis propria) layers and ranged from 6 to $25 \mathrm{~mm}$ in size (median size: $13 \mathrm{~mm}$ ) ( $\triangleright$ Table 2 ). All lesions showed mural growth pattern and hypoechoic echogenicity. Thirty-four lesions (34/ $36,94 \%)$ were heterogeneous and the borders were indistinct in 32 lesions (32/36, 89\%). Anechoic duct-like structures appeared in 32 lesions (32/36, 89\%). Two lesions involved only one sonographic layer of the gastric wall: the third layer. Twenty-eight lesions involved two sonographic layers: 13 in the second and third layers and 15 in the third and fourth layers. Three lesions involved the second, third, and fourth layers, and three involved the third, fourth, and fifth layers. On the basis of the sonographic layer of origin, 15 of 36 lesions were S-type and the other 21 lesions were D-type.

On ME-NBI, a microscopic opening was identified in 22 of 27 lesions $(81 \%)$ in which a macroscopic opening was not observed during conventional endoscopy ( $>$ Table3) ( Fig.2). As a result, macroscopic or microscopic opening was observed in 31 lesions $(31 / 36,86 \%)$. Of 32 lesions in which anechoic duct-like structures appeared on EUS, 29 lesions had a macroscopic or microscopic opening on ME-NBI. On the contrary, of four lesions in which anechoic duct-like structures did not appear on EUS, two lesions had a macroscopic or microscopic opening. Therefore, frequency of macroscopic or microscopic opening was higher in lesions with anechoic duct-like struc- tures than that in lesions without anechoic duct-like structures on EUS (91\% [29/32] vs 50\% [2/4], P=0.027). Of 21 D-type lesions, 19 lesions had a macroscopic or microscopic opening on $\mathrm{ME}-\mathrm{NBI}$, and of 15 S-type lesions, 12 lesions had a macroscopic or microscopic opening. No difference was observed in the frequency of macroscopic or microscopic opening between S-type and D-type lesions (80\% [12/15] vs $90 \%$ [19/21], $P=0.370)$. In addition, focal loss of microsurface structure and presence of a thickened submucosal vessel were observed in six $(6 / 36,17 \%)$ and five lesions $(5 / 36,14 \%)$, respectively.

\section{Discussion}

A firm round or oval subepithelial lesion with central dimpling or umbilication, which corresponds to the opening of a duct, is the typical endoscopic finding for heterotopic pancreas [7]. However, it is seen in only about one-third of cases $[5,8]$. Therefore, we used ME-NBI to identify any additional data to predict diagnosis of heterotopic pancreas. We found that a microscopic opening was present on ME-NBI in more than $80 \%$ of lesions without a macroscopic opening. To our knowledge, this study is the first to report about ME-NBI for gastric heterotopic pancreas.

ME-NBI is a powerful diagnostic modality because it can enable visualization of real-time microscopic images of the mucosal surface [9], and the most advanced endoscope has a maximal resolution power as small as $6.4 \mu \mathrm{m}$ [10]. Although the clinical usefulness of ME-NBI has been reported in gastritis and early gastric cancer $[11,12]$, reports on use of ME-NBI in gastric subepithelial lesions are few. Histologically, heterotopic pancreas mainly consists of exocrine tissue and excretory ducts. In a case series including 32 cases of heterotopic pancreas in the gastrointestinal tract, all had excretory ducts and $97 \%$ had acini [13]. As previously mentioned, the small ducts mainly drain directly into the gastric lumen. In the current study, a microscopic opening was identified in 22 of 27 heterotopic pancreas lesions without a macroscopic opening. These findings suggest the usefulness of ME-NBI to predict diagnosis of heterotopic pancreas.

Is a microscopic opening present only in heterotopic pancreas? Based on our experience, we could detect a microscopic opening in about one-third of gastritis cystica profunda cases (unpublished data). However, because gastritis cystica profunda has a soft consistency due to its inner liquid component (positive pillow sign), it can be easily differentiated from heterotopic pancreas, which has a hard consistency.

We also found a focal loss of microsurface structure in six lesions. The focal loss of microsurface is thought to be caused by chronic irritation from enzymes secreted by the heterotopic pancreas. In addition, a thickened submucosal vessel was noted in five lesions. The appearance of this vessel in gastric subepithelial lesions was previously reported in a case of gastric carcinoid tumor [14]. Therefore, we speculate that presence of a thickened submucosal vessel is an indirect sign suggesting that a lesion has subepithelial components, such as endocrine nests in the carcinoid tumor or pancreas acinar nest in the heterotopic pancreas. There remains a paucity of data about ME- 


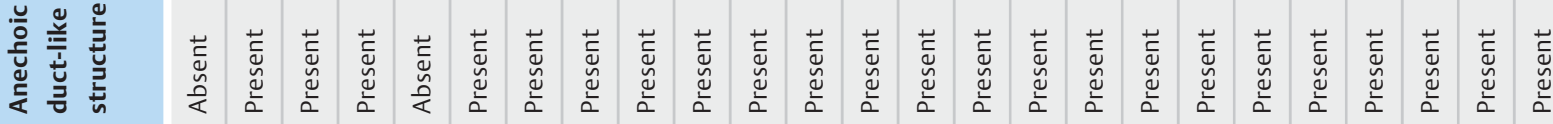

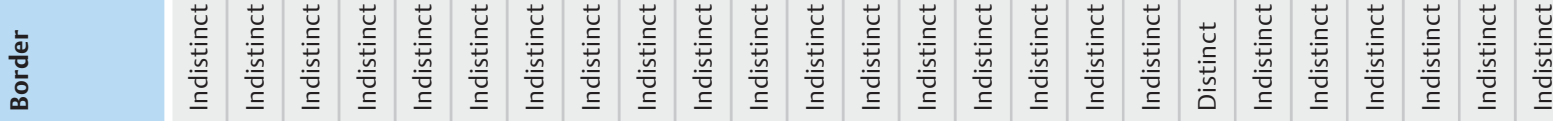
点

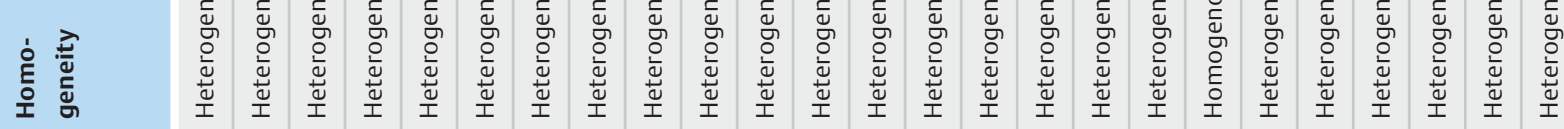

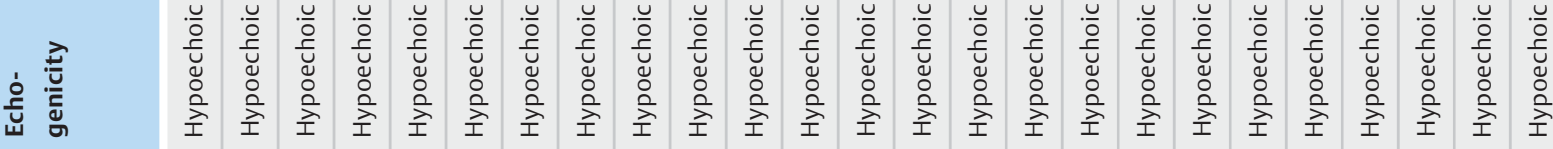

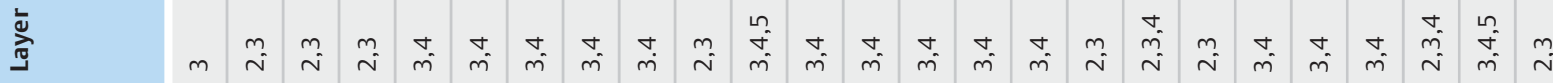

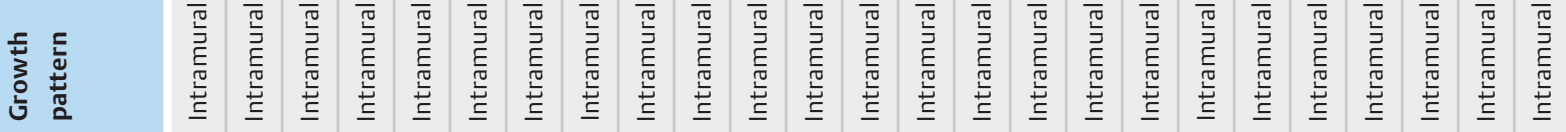

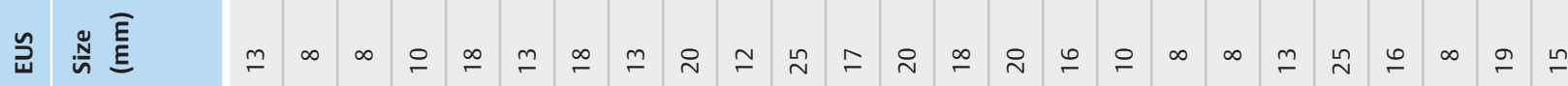

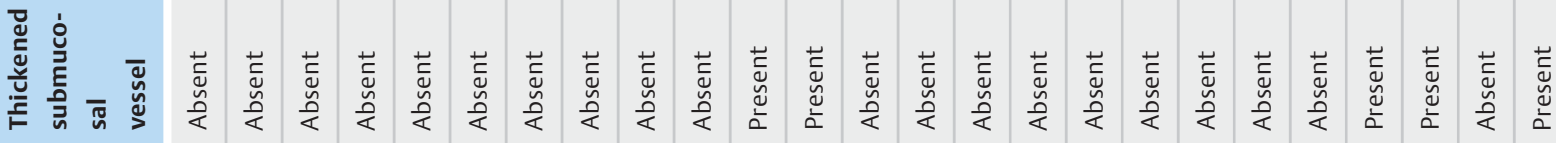

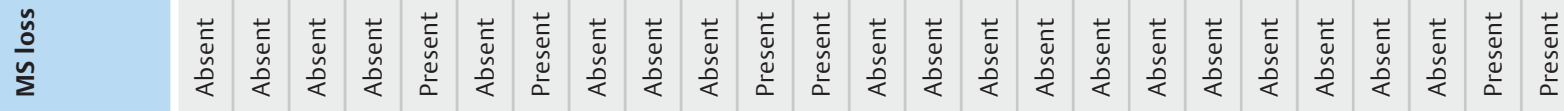

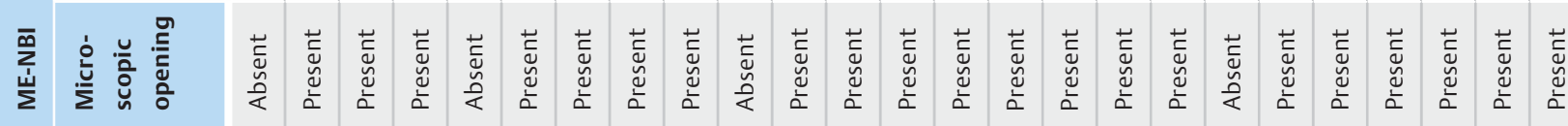

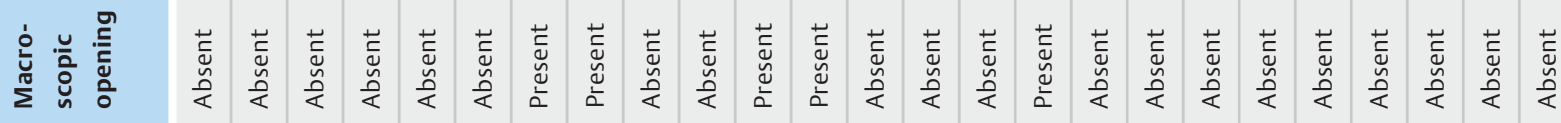

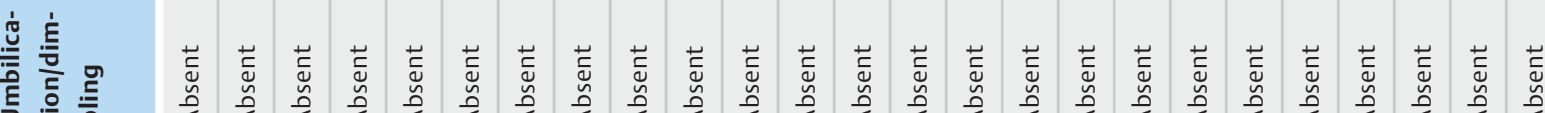
츤 E气

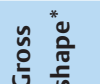




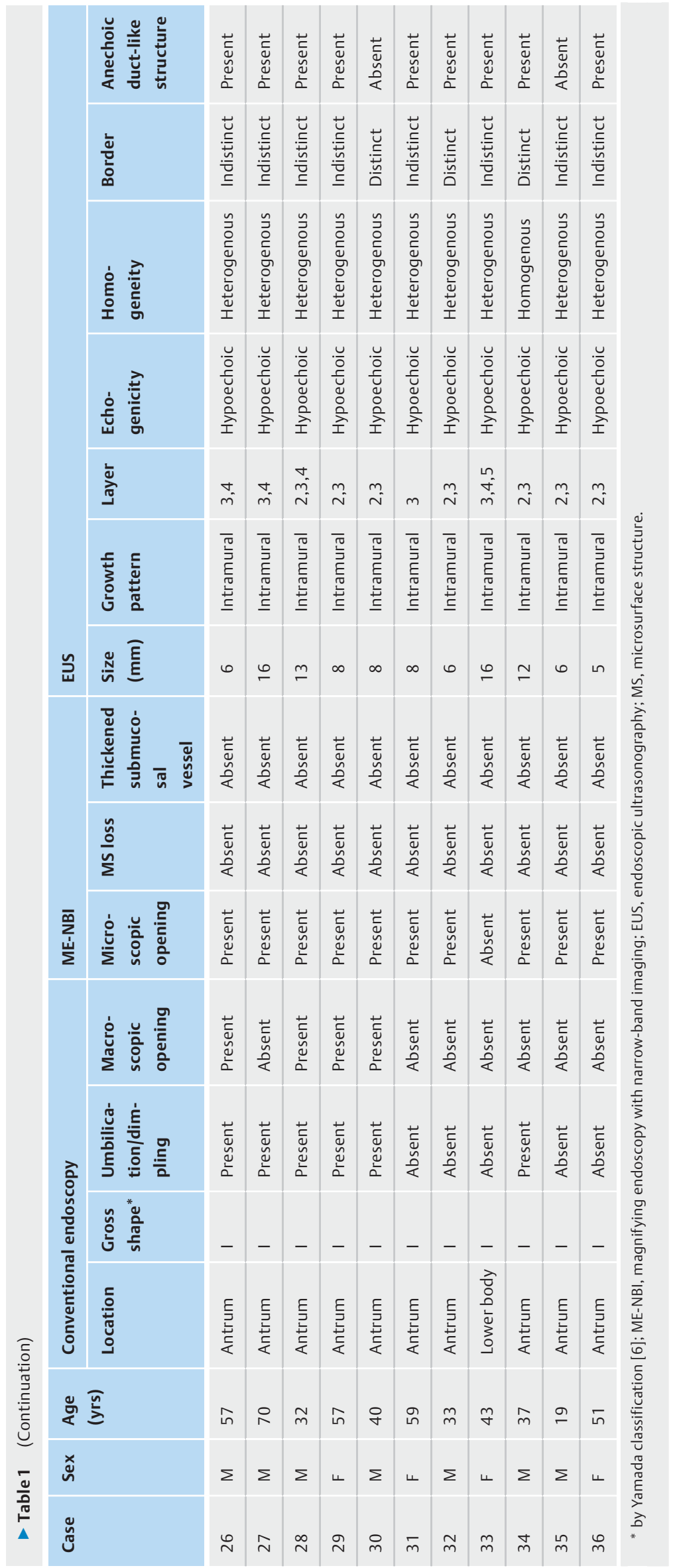


- Table 2 Baseline and endosonographic characteristics of 36 patients with gastric heterotopic pancreas.

\begin{tabular}{l|l|}
\hline $\begin{array}{l}\text { Patient characteristics } \\
\text { Median age (years, range) }\end{array}$ & $40(15-70)$ \\
\hline Sex, n (\%) & $22(61)$ \\
\hline - Male & $14(39)$ \\
\hline - Female
\end{tabular}

Endoscopic characteristics

Location, $\mathrm{n}(\%)$

\begin{tabular}{|l|r|}
\hline - Antrum & $30(83)$ \\
\hline - Lower body & $4(11)$ \\
\hline - Mid-body & $2(6)$ \\
\hline Macroscopic shape, $\mathrm{n}(\%)$ & \\
\hline - Yamada type $\mathrm{I}$ & $36(100)$ \\
\hline Umbilication/dimpling, $\mathrm{n}(\%)$ & \\
\hline - Present & $6(17)$ \\
\hline - Absent & $30(83)$ \\
\hline Opening, $\mathrm{n}(\%)$ & \\
\hline - Present & $9(25)$ \\
\hline - Absent & $27(75)$ \\
\hline
\end{tabular}

\section{Endosonographic characteristics}

$\begin{array}{ll}\text { Median size (mm, range) } & 13(6-25)\end{array}$

Growth pattern, n (\%)

\begin{tabular}{|l|r|}
\hline - Mural & $36(100)$ \\
\hline Echogenicity, $\mathrm{n}(\%)$ & $36(100)$ \\
\hline - Hypoechoic & \\
\hline Homogeneity, $\mathrm{n}(\%)$ & $34(94)$ \\
\hline - Heterogenous & $2(6)$ \\
\hline - Homogenous & \\
\hline Border, $\mathrm{n}(\%)$ & $32(89)$ \\
\hline - Indistinct & $4(11)$ \\
\hline - Distinct & \\
\hline Anechoic duct-like structure, $\mathrm{n}(\%)$ & $32(89)$ \\
\hline - Present & $4(11)$ \\
\hline - Absent & \\
\hline Endosonographic classification, $\mathrm{n}(\%)$ & $15(42)$ \\
\hline - Superficial type & $21(58)$ \\
\hline - Deep type & \\
\hline
\end{tabular}




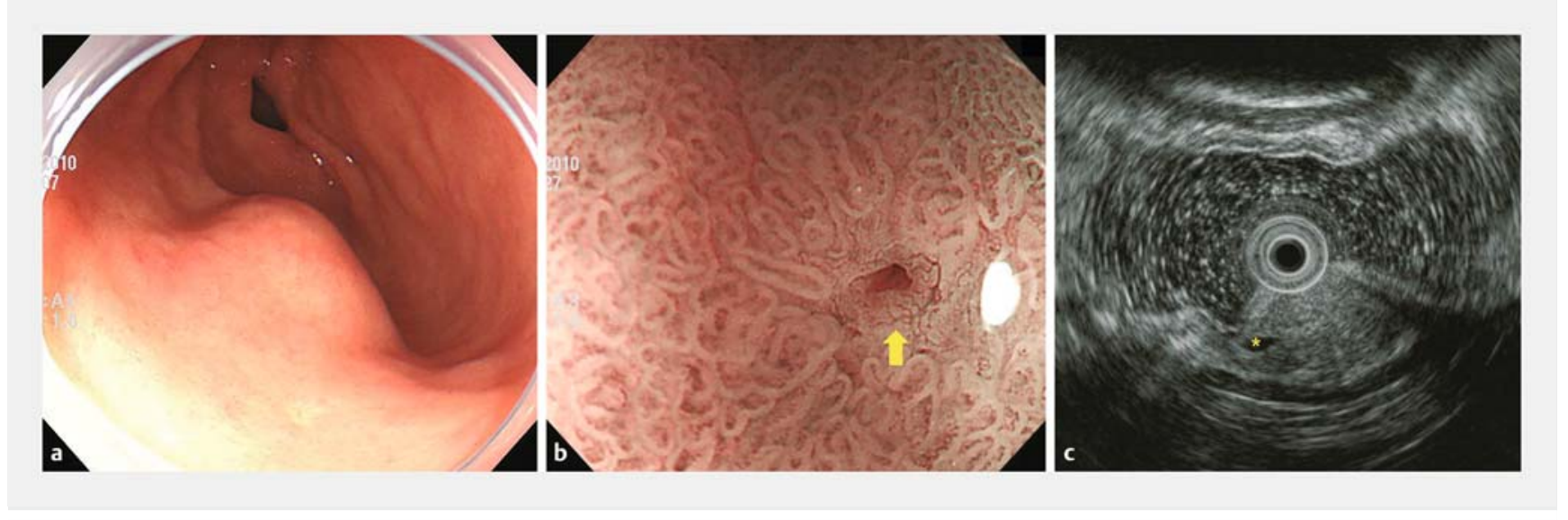

- Fig. 2 A representative case of heterotopic pancreas (case 31). a A subepithelial lesion is seen on the greater curvature of the gastric antrum. b On magnifying endoscopy with narrow-band imaging, a microscopic opening is observed on its surface (arrow). c Endosonographic image obtained with a 20-MHz catheter probe. A heterogeneously hypoechoic lesion with lobular margins is located within the submucosal layer. Anechoic duct-like structure is observed at its periphery (asterisk).

\section{Conclusion}

In conclusion, the characteristic ME-NBI feature of heterotopic pancreas is presence of a microscopic opening on its surface. This ME-NBI feature is potentially useful for differentiating heterotopic pancreas from other subepithelial tumors in the stomach.

\section{Acknowledgements}

This study was supported by a grant from the National R\&D Program for Cancer Control, Ministry for Health, Welfare and Family Affairs, Republic of Korea (0920050) and by the Medical Research Center Program through the National Research Foundation of Korea grant funded by the Korea government (NRF2015R1A5A2009656).

\section{Competing interests}

None

\section{References}

[1] DeBord JR, Majarakis JD, Nyhus LM. An unusual case of heterotopic pancreas of the stomach. Am J Surg 1981; 141: 269-273

[2] Burke GW, Binder SC, Barron AM et al. Heterotopic pancreas: gastric outlet obstruction secondary to pancreatitis and pancreatic pseudocyst. Am J Gastroenterol 1989; 84: 52 - 55
[3] Ryu DY, Kim GH, Park doY et al. Endoscopic removal of gastric ectopic pancreas: An initial experience with endoscopic submucosal dissection. World ] Gastroenterol 2010; 16: 4589-4593

[4] Hsia CY, Wu CW, Lui WY. Heterotopic pancreas: a difficult diagnosis. J Clin Gastroenterol 1999; 28: 144-147

[5] Park SH, Kim GH, Park DY et al. Endosonographic findings of gastric ectopic pancreas: a single center experience. J Gastroenterol Hepatol 2011; 26: 1441 - 1446

[6] Yamada T, Ichikawa H. X-ray diagnosis of elevated lesions of the stomach. Radiology 1974; 110: 79-83

[7] Chen SH, Huang WH, Feng CL et al. Clinical analysis of ectopic pancreas with endoscopic ultrasonography: an experience in a medical center. J Gastrointest Surg 2008; 12: 877-881

[8] Ormarsson OT, Gudmundsdottir I, Marvik R. Diagnosis and treatment of gastric heterotopic pancreas. World J Surg 2006; 30: 1682-1689

[9] Yao K. Clinical application of magnifying endoscopy with narrownand imaging in the stomach. Clin Endosc 2015; 48: 481-490

[10] Yao K, Anagnostopoulos GK, Ragunath K. Magnifying endoscopy for diagnosing and delineating early gastric cancer. Endoscopy 2009; 41: $462-467$

[11] An JK, Song GA, Kim GH et al. Marginal turbid band and light blue crest, signs observed in magnifying narrow-band imaging endoscopy, are indicative of gastric intestinal metaplasia. BMC Gastroenterol 2012; 12: 169

[12] Ok KS, Kim GH, Park doY et al. Magnifying endoscopy with narrow band imaging of early gastric cancer: correlation with histopathology and mucin phenotype. Gut Liver 2016; 10: 532 - 541

[13] Pang LC. Pancreatic heterotopia: a reappraisal and clinicopathologic analysis of 32 cases. South Med J 1988; 81: 1264-1275

[14] Singh R, Yao K, Anagnostopoulos G et al. Microcarcinoid tumor diagnosed with high-resolution magnification endoscopy and narrow band imaging. Endoscopy 2008; 40: (Suppl. 02): E12 